\title{
Utilization of mango peel extracts on the biodegradable films for active packaging
}

\begin{abstract}
Mango peels extract (MPE) was incorporated into fish gelatin films to determine their physical, barrier, mechanical and antioxidant properties for active food packaging. Films with three different concentrations of MPE (1-5\%) were prepared by solution casting method. Films incorporated with MPE showed a decrease $(\mathrm{P}>0.05)$ of water vapor permeability (WVP) and lower $(\mathrm{P} \leq 0.05)$ films solubility. High level of MPE films also exhibited more rigid and less flexible film formation. Colored tint films and a reduction in transparency were due to the hydrogen bond linkages between fish gelatin molecules and phenolic content within the film matrix. Higher free radicals scavenging activities also observed for films with higher concentrations of MPE. This study reveals the benefits of mango by-products incorporated into gelatin based films as a potential material for active packaging.
\end{abstract}

Keyword: Mango peels; Agricultural waste; Biodegradable films; Gelatin; Active packaging 